\title{
Determinação do Teor de NR/SBR em Misturas: Associação de Dados DTG e FT-IR
}

\author{
Rita C. L. Dutra, Milton F. Diniz, Ana P. Ribeiro, Vera L. Lourenço, Silvana N. Cassu, Margarete F. P. Azevedo \\ Divisão de Química, IAE, CTA, SP
}

\begin{abstract}
Resumo: Misturas contendo teor conhecido de borracha natural (NR) e copolímero de butadieno e estireno (SBR) foram preparadas nos laboratórios do CTA e do IFOCA como amostras de referência para a elaboração de uma curva analítica, visando à determinação do teor de NR e SBR por análise pirólise/FT-IR. Termogravimetria foi usada para determinar o teor real de NR e SBR nas misturas por meio da razão entre as alturas dos picos da curva derivada (DTG). As bandas FTIR escolhidas na região MIR foram $885 \mathrm{~cm}^{-1}\left(\mathrm{~A}_{1}\right)$ para NR e $699 \mathrm{~cm}^{-1}\left(\mathrm{~A}_{2}\right)$ para SBR. Os valores de absorvância relativa $\left(\mathrm{A}_{1} / \mathrm{A}_{2}\right)$ versus a relação NR/SBR, obtida a partir dos dados fornecidos pela análise $\mathrm{DTG}$, foram usados para construir uma curva com uma boa correlação linear $(\mathrm{R}=0,998)$, a qual possibilita determinar os teores de NR e SBR em misturas onde os componentes têm a mesma composição química.
\end{abstract}

Palavras-chave: Borracha natural (NR), copolímero de butadieno e estireno (SBR), mistura, FT-IR, termogravimetria.

\section{Determination of NR/SBR Content in Blends: Combining DTG and FT-IR Data}

Abstract: Blends with known contents of natural rubber (NR) and butadiene-styrene copolymer (SBR) were prepared at CTA and IFOCA laboratories as sample references for producing an analytical curve to determine the contents of NR and SBR via pyrolysis/FT-IR analysis. Thermogravimetry was used to quantify the real content of NR and SBR in the blends through the ratio between the intensity of the peaks in the derivative curve (DTG). The FT-IR analytical bands chosen at MIR region were $885 \mathrm{~cm}^{-1}\left(\mathrm{~A}_{1}\right)$ for NR and $699 \mathrm{~cm}^{-1}\left(\mathrm{~A}_{2}\right)$ for SBR. Values of relative absorbance $\left(\mathrm{A}_{1} / \mathrm{A}_{2}\right)$ versus the NR/ SBR contents data obtained from DTG analysis were used to construct an analytical curve with a good linear correlation $(\mathrm{R}=0.998)$ which allows one to determine contents of NR and SBR in blends of similar composition.

Keywords: Natural rubber (NR), butadiene-styrene copolymer (SBR), blends, FT-IR, thermogravimetry.

\section{Introdução}

A espectroscopia no infravermelho (IR) pode ser utilizada para a identificação inequívoca da maioria dos elastômeros e suas misturas ${ }^{[1-3]}$ por meio da análise dos espectros IR de seus pirolisados. No entanto, a quantificação destes componentes requer a elaboração de uma curva que relacione a intensidade da banda analítica à concentração do elastômero.

Segundo Gedeon ${ }^{[1]}$, Smithers Scientific Services analisaram por IR constituintes elastoméricos em composições curadas de borracha, desde 1963, usando técnica de pirólise. Cálculos de intensidades e sua proporção (absorvância relativa) eram feitos à mão. Um gráfico da relação de bandas e porcentagem de composição era feito e este gráfico, então, usado para calcular a composição de amostras desconhecidas por meio dos valores de absorvância relativa. Este método era demorado e apresentava grandes possibilidades de erro devido aos numerosos cálculos envolvidos.

Vinte anos depois, Gedeon e colaboradores ${ }^{[1]}$ fizeram uma revisão desse procedimento desenvolvido. O objetivo foi estudar e melhorar a precisão da análise e diminuir o tempo necessário para sua execução. A revisão incluiu o uso de um computador para coletar os dados dos espectros IR de misturas de borracha natural (NR), copolímero de butadieno e estireno (SBR) e polibutadieno (BR), calcular a intensidade ou área das bandas características, obter as proporções necessárias, e comparar os resultados aos obtidos para amostras conhecidas. Foi concluído, como esperado, que o uso do computador permitiu a obtenção de dados mais precisos e em menor tempo de análise, e que os valores obtidos por medidas de intensidade de bandas são mais precisos que os obtidos por área. Entretanto, nenhuma outra metodologia foi utilizada para fornecer a concentração de cada elastômero na mistura.

Em estudo sobre misturas NR/SBR feito anteriormente por nosso grupo de espectroscopia IR no IFOCA (França $)^{[4]}$, foi obtida uma curva analítica com boa correlação linear $(\mathrm{R}=0,994)$ utilizando-se os teores nominais, obtenção do pirolisado num forno a $575 \pm 5^{\circ} \mathrm{C}$ e valores de intensidade de bandas para cálculo de absorvância relativa. $\mathrm{O}$ desvio relativo da metodologia ficou entre 5 e $8 \%$, que, embora alto, foi considerado aceitável paras as condições utilizadas. Como nenhuma outra metodologia foi utilizada

Autor para correspondência: Rita C. L. Dutra, AQI, CTA, IAE, Pr. Mal Eduardo Gomes 50, CEP: 12228-904, São José dos Campos, SP. E-mail:ritad@iae.cta.b 
para validação, imaginou-se, então, que técnicas de análise térmica pudessem ser utilizadas para compor nova metodologia, visando maior precisão.

É conhecida a utilização da calorimetria exploratória diferencial (DSC) para identificação de elastômeros e suas misturas, sendo que as curvas de degradação térmica e termooxidativa podem ser usadas como impressão digital para identificá-los ${ }^{[5-8]}$. A associação destas curvas com a determinação da temperatura de transição vítrea ou de fusão também tem sido de grande ajuda na identificação de elastômeros e suas misturas ${ }^{[5]}$.

A termogravimetria (TG), por usar um programa de aquecimento dinâmico reprodutível e assim permitir o controle fino da história térmica da amostra, tem sido bastante usada para a análise de elastômeros ${ }^{[5-10]}$. A curva de perda de massa TG possibilita que o teor relativo dos componentes orgânicos, incluindo óleo de processamento, plastificantes, elastômeros e negro de fumo, e inorgânicos, como cargas e cinzas, possa ser facilmente calculado. A curva termogravimétrica derivada em tempo versus a temperatura (DTG) facilita a identificação e quantificação de elastômeros e suas misturas, pois, enquanto que os componentes separadamente possam degradar numa mesma faixa de temperatura, muito provavelmente eles perderão massa com diferentes velocidades, originando vários máximos na curva DTG, o que permite uma melhor definição das etapas de decomposição, fornecendo por vezes, também um perfil característico dos elastômeros ${ }^{[6,7,10]}$.

Uma análise cuidadosa dos picos DTG permite diferenciar entre os vários elastômeros e os óleos de processamento e plastificantes mais voláteis. Sircar e Lamond ${ }^{[5]}$ aplicaram a técnica DTG para estudar as bandas de rolagem e as laterais de pneus. NR, SBR e BR foram caracterizados pela temperatura de seus picos DTG, o que foi então usado para identificá-los nas misturas utilizadas nos pneus. Brazier ${ }^{[6]}$ estudou cuidadosamente as características das curvas DTG de diferentes tipos de NR, BR e SBR e suas misturas concluindo que as temperaturas dos picos DTG das misturas correspondiam diretamente aos valores registrados para os componentes puros, e que a altura do pico estava relacionada ao teor do componente, embora, a relação linear observada para a mistura NR/BR somente fosse válida na faixa 80:20 a 20:80. Misturas contendo SBR, NR, EPDM e BR utilizadas em pneus, foram caracterizadas pelas técnicas DSC e TG/ DTG por Sircar ${ }^{[7]}$ e Lourenço e Dutra ${ }^{[8]}$. Obviamente, a sobreposição da decomposição térmica dos dois componentes elastôméricos pode ocorrer devido à sua estabilidade térmica similar, o que pode causar uma alteração na posição dos picos DTG.

Yang, Kaliaguine e Roy ${ }^{[10]}$ analisaram quantitativamente misturas binárias de elastômeros e óleo de processamento utilizando curvas DTG simuladas a partir dos parâmetros cinéticos obtidos das curvas DTG de NR, SBR, BR isolados. No entanto, este método pressupõe que não haja interação entre os componentes e necessita das curvas desses polímeros isolados, além de uma série de simulações e comparações com a curva experimental.
A associação do mérito qualitativo da espectroscopia IR ao quantitativo da análise DTG para diferentes materiais tem apresentado bons resultados ${ }^{[8,11,12]}$.

Mediante o exposto, e desde que, atualmente, estão disponíveis em nossos laboratórios, no Brasil, pirolisador com melhor controle de temperatura e analisador térmico TG para fornecer a concentração do elastômero nas misturas, resolveu-se refazer o estudo para verificar se melhores resultados poderiam ser obtidos.

\section{Experimental}

NR (crepe branco cedido por Elastic S/A) e SBR (1502, Petroflex) foram utilizados para preparar as misturas NR/SBR: 10/90; 20/80*; 30/70; 50/50*; 70/30; 80/20* e 90/10 conforme a formulação (phr e \%) mostrada na Tabela 1 . As misturas assinaladas com * foram feitas no CTA e não contêm carbonato de cálcio, enquanto que, as demais foram feitas no IFOCA. Após mistura em misturador de rolos, foram moldadas sob pressão em placa de espessura $1 \mathrm{~mm}$ a $160^{\circ} \mathrm{C}$ por $20 \mathrm{~min}$, tempo ótimo fornecido pelo reograma da mistura. As análises DTG foram feitas numa termobalança TGA-951 Du Pont, $30 \mathrm{mg}$ em porta-amostras de platina, sob nitrogênio, a $10{ }^{\circ} \mathrm{C} / \mathrm{min}$, da T amb. até $600^{\circ} \mathrm{C}$, em duplicata. $300 \mathrm{mg}$ das amostras, num único pedaço, foram extraídas em acetona e pirolisados no pirolisador SPECTRA-TECH PY-2, a $580{ }^{\circ} \mathrm{C}$, por $2 \mathrm{~min}$. Os pirolisados foram imediatamente analisados no espectrômetro FT-IR 1750 PERKINELMER (resolução $4 \mathrm{~cm}^{-1}$, ganho 1, 4000 a $400 \mathrm{~cm}^{-1}, 40$ varreduras) como filmes líquidos com espaçador de $0,025 \mathrm{~mm}$.

\section{Resultados e Discussão}

\section{Análises DTG}

A Figura 1 mostra as curvas DTG obtidas para os elastômeros NR e SBR. A perda de massa ocorrida até $200{ }^{\circ} \mathrm{C}$ foi atribuída

Tabela 1. Formulação das misturas de elastômeros estudadas

\begin{tabular}{lccc}
\hline \multicolumn{1}{c}{ Ingrediente } & phr & \%* & \%** \\
\hline NR/SBR & 100 & 67,9 & 58,0 \\
ZnO (Ativador) & 5 & 3,4 & 2,9 \\
Ácido Esteárico (Ativador) & 1 & 0,7 & 0,6 \\
Negro de fumo N330 (carga) & 30 & 20,4 & 17,4 \\
Carbonato de cálcio EXH1 (carga) & 25 & - & 14,5 \\
Plastificante 729FC & 5 & 3,4 & 2,9 \\
S(agente de vulcanização) & 2,5 & 1,7 & 1,5 \\
CBS (acelerador) & 0,8 & 0,5 & 0,5 \\
6PPD (Agente protetor) & 1,5 & 1,0 & 0,9 \\
Permanax TQ (Agente protetor) & 1,5 & 1,0 & 0,9 \\
\hline *CTA: não contêm carbonato de cálcio **IFOCA & &
\end{tabular}


à eliminação do plastificante e agentes de processamento e outros componentes de baixo ponto de ebulição.

NR apresenta um pico intenso em $389^{\circ} \mathrm{C}$ seguido por um ombro em $443{ }^{\circ} \mathrm{C}$, representando a maior parte da perda de massa, sendo por isso, atribuído à decomposição do elastômero. $\mathrm{O}$ formato deste pico é característico do $\mathrm{NR}^{[7,9,10]}$, porém, o máximo ocorre em temperatura mais alta que descrito na literatura $\left(334-384^{\circ} \mathrm{C}\right)^{[6,9,10]}$, o que mostra que usar a temperatura de pico fornecida pela literatura pode levar a erros, sendo mais seguro, sempre que possível, utilizar dados obtidos a partir de elastômeros de fontes e tipos semelhantes.

O SBR-1502 utilizado contém $23 \%$ de estireno, sendo obtido em emulsão contendo sabões resinosos e derivados de ácido graxo, coagulado por sal-ácido (5-7\%) e apresentou um pico DTG entre 180 e $330^{\circ} \mathrm{C}$, pouco intenso (Figura 1), relativo à perda de massa de $6,5 \%$. Isto concorda com o observado por Brazier ${ }^{[6]}$, segundo o qual SBR obtido por emulsão pode apresentar perda de massa já em $175^{\circ} \mathrm{C}$, a qual depende do tipo de emulsificante utilizado.

Na Figura 1 pode ser observado que o SBR apresenta um ombro em $389{ }^{\circ} \mathrm{C}$ seguido por um pico intenso em $468{ }^{\circ} \mathrm{C}$ devido à decomposição do polímero. $\mathrm{O}$ ombro tem origem na despolimerização dos segmentos polibutadiênicos do SBR, originando predominantemente butadieno e vinilciclohexeno ${ }^{[6]}$. O pico resulta da degradação simultânea do estireno e butadieno, razão pela qual a identificação de SBR em presença de BR é dificultada ${ }^{[6]}$. A temperatura do máximo deste pico depende do teor de estireno presente no SBR, cujo aumento a desloca para temperaturas mais baixas. A exotermicidade da degradação do polibutadieno diminui a reprodutibilidade da altura dos picos. A ocorrência de dois máximos, com o primeiro mais intenso, é possível, embora a formulação possa obscurecê-1 ${ }^{[9]}$. Neste caso, isto não foi observado, embora, um pequeno, mas, repetitivo ombro ocorra na região de $435{ }^{\circ} \mathrm{C}$, confirmando o perfil relatado por Brazier $^{[6]}$.

A comparação das curvas NR e SBR na Figura 1 mostra que o pico DTG do NR está praticamente completo antes do máximo do pico principal do SBR, indicando que NR deve contribuir pouco na intensidade do pico DTG do SBR, como observado por Brazier e Nicke ${ }^{[9]}$. Ambos, em nitrogênio, apresentam degradação quase completa, sem carbonizar, restando

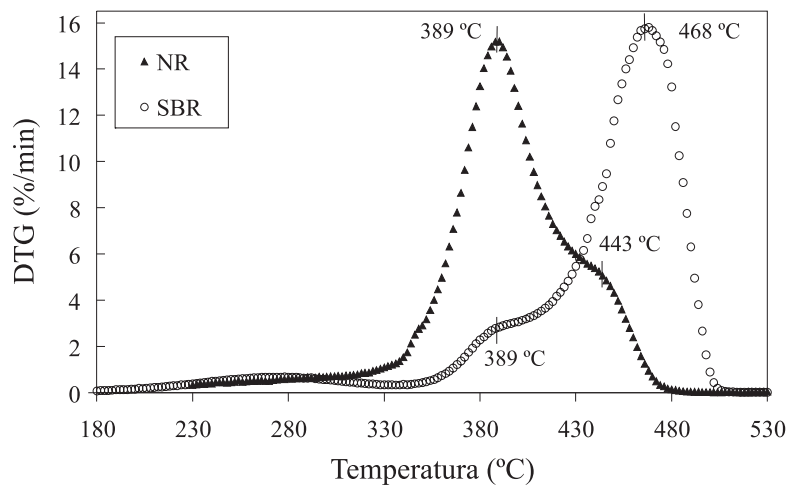

Figura 1. Curvas DTG de NR, SBR, (nitrogênio, $10^{\circ} \mathrm{C} / \mathrm{min}$ ).

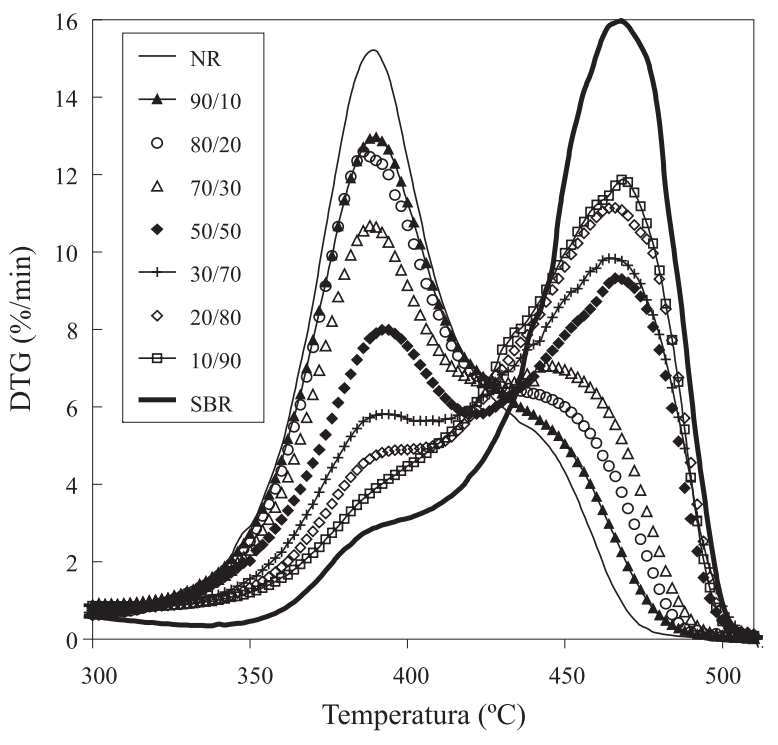

Figura 2. Curvas DTG de NR, SBR e suas misturas, (nitrogênio, $10{ }^{\circ} \mathrm{C} /$ $\min )$

menos de $1 \%$ da massa inicial, de forma que o resíduo, nas amostras formuladas, é composto por negro de fumo além das cargas e resíduos inorgânicos. Foi observado que a presença de NF e a vulcanização não afetam a posição da curva DTG do NR ${ }^{[9]}$.

As curvas DTG obtidas para as misturas NR/SBR estão na Figura 2 onde pode ser observado que exibem um pico DTG com máximo em temperatura próxima a $(389 \pm 3)^{\circ} \mathrm{C}$, cuja intensidade $\left(h_{1}\right)$ acompanha o teor em NR, e outro pico entre 420 e $500{ }^{\circ} \mathrm{C}$, cuja temperatura do máximo e sua intensidade $\left(\mathrm{h}_{2}\right)$ aumentam com o aumento do teor em SBR na mistura. Em muitas misturas estudadas ${ }^{[6]}$ este parece ser um fenômeno comum, com a estabilidade do componente mais estável sendo reduzida, o que pode ser atribuído ao fato dos radicais produzidos na degradação do elastômero menos estável iniciarem a degradação do mais estável.

A partir das curvas DTG obtidas para as misturas NR/ SBR (Figura 2) foram coletados os valores das temperaturas dos máximos dos picos DTG correspondentes à eliminação dos elastômeros $\left(\mathrm{T}_{1}\right.$ e $\left.\mathrm{T}_{2},{ }^{\circ} \mathrm{C}\right)$, mostrados na Tabela 2, sua intensidade $\left(h_{1}\right.$ e $\left.h_{2}, \% / m i n\right)$ e calculada a relação $h_{1} / h_{2}$.

Os valores de $h_{1}$ e $h_{2}$ obtidos inicialmente não apresentaram boa correlação linear com os respectivos teores de NR ou SBR. No entanto, foi observado que a correlação melhorava quando eram consideradas apenas misturas de mesma fonte, ou seja, IFOCA ou CTA. A diferença entre elas está no teor de resíduo, devido à presença ou não de carbonato de cálcio. Os valores de $h_{1}$ e $h_{2}$ originais foram, então, corrigidos desprezando-se o resíduo obtido (R), ou seja:

$$
\mathrm{h}_{\mathrm{c}}=\mathrm{h}_{\mathrm{i}} \cdot 100 /(100-\mathrm{R})
$$

sendo que os valores corrigidos são os mostrados na Tabela 2. Desta forma, foi obtida uma boa correlação linear de $h_{1}$ com o teor de $\mathrm{NR}(\mathrm{R}=0,99)$, porém, o mesmo não ocorreu com $\mathrm{h}_{2}$ e o teor de SBR $(\mathrm{R}=0,96)$.

Foi, então, avaliado o comportamento da relação $h_{1} / h_{2}$ com o 
Tabela 2. Dados DTG das misturas de NR/SBR

\begin{tabular}{cccccc}
\hline Amostra & $\begin{array}{c}\mathbf{T}_{\mathbf{1}} \\
\left({ }^{\mathbf{}} \mathbf{C}\right)\end{array}$ & $\begin{array}{c}\mathbf{h}_{\mathbf{1}} \\
(\boldsymbol{\%} / \mathbf{m i n})\end{array}$ & $\begin{array}{c}\mathbf{T}_{\mathbf{2}} \\
\left({ }^{\mathbf{C}} \mathbf{C}\right)\end{array}$ & $\begin{array}{c}\mathbf{h}_{\mathbf{2}} \\
(\boldsymbol{\%} / \mathbf{m i n})\end{array}$ & $\mathbf{h}_{\mathbf{1}} / \mathbf{h}_{\mathbf{2}}$ \\
\hline NR & 388,6 & 15,23 & 443,5 & 5,11 & 2,98 \\
SBR & 388,8 & 2,84 & 460,3 & 15,99 & 0,18 \\
NR/SBR & 389,2 & 13,04 & 447,5 & 5,30 & 2,46 \\
$90 / 10$ & & & & & \\
80/20 & 386,4 & 12,62 & 450,2 & 5,99 & 2,11 \\
$70 / 30$ & 389,0 & 10,68 & 446,4 & 7,04 & 1,52 \\
$50 / 50$ & 393,0 & 8,00 & 467,9 & 9,31 & 0,86 \\
$30 / 70$ & 390,5 & 5,80 & 465,3 & 9,89 & 0,59 \\
$20 / 80$ & 387,2 & 4,60 & 465,4 & 11,14 & 0,41 \\
$10 / 90$ & 384,7 & 3,51 & 468,8 & 11,88 & 0,30 \\
\hline
\end{tabular}

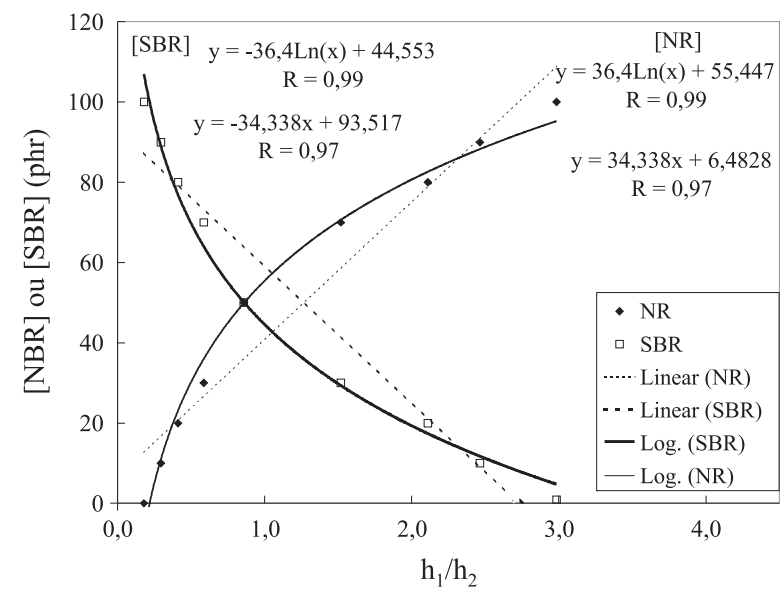

Figura 3. Variação de h1/h2 (DTG) com o teor de NR e SBR nas misturas

teor de NR ou SBR. A Figura 3 mostra que o ajuste linear é menos satisfatório que o ajuste logarítmico, o qual tem melhor fator de correlação $(\mathrm{R}=0,99)$. É interessante observar que não é necessário corrigir quanto ao teor de resíduo pois o valor de $\mathrm{h}_{1} /$ $\mathrm{h}_{2}$ não é afetado por esta correção, de forma que composições com resíduos diferentes podem ser comparadas. Desta forma, a equação do ajuste logarítmico foi usada para calcular os teores em NR e SBR e a relação [NR]/[SBR] mostrada na Tabela 3.

\section{Análise FT-IR}

A Figura 4 inclui os espectros FT-IR de algumas misturas de NR/SBR estudadas. As bandas analíticas escolhidas, 885 $\mathrm{cm}^{-1}\left(\mathrm{~A}_{1}\right)$ para NR e $699 \mathrm{~cm}^{-1}\left(\mathrm{~A}_{2}\right)$ para $\mathrm{SBR}$, são atribuídas, respectivamente, aos grupos vinilideno $\left(\omega \mathrm{CH}_{2}\right)$ e $\delta \mathrm{C}-\mathrm{H}$ (substituição aromática). A linha de base para a banda de NR foi traçada de 940 a $840 \mathrm{~cm}^{-1}$ e para SBR, de 740 a $680 \mathrm{~cm}^{-1}$.

Com base na lei de Lambert-Beer, e em concordância com o observado por Gedeon e colaboradores ${ }^{[1]}$, um entendimento das limitações do uso de pirólise IR para análise quantitativa deve ser feito. Para aplicar a lei de de Lambert-Beer a esta análise, os dados devem ser colocados em função da

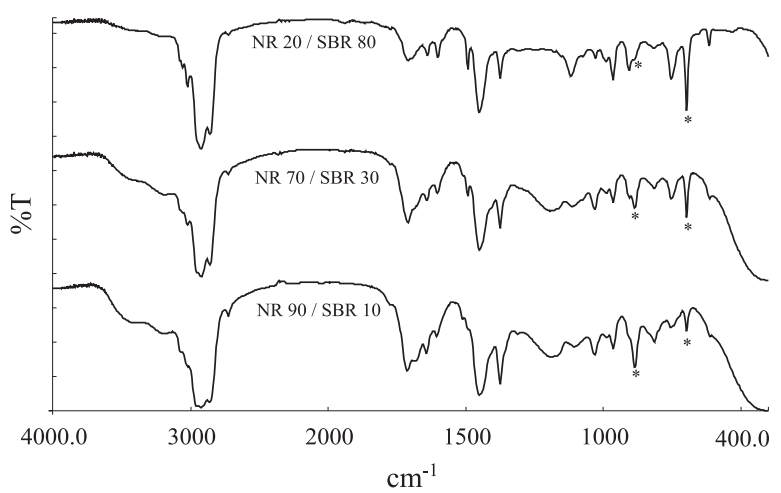

Figura 4. Espectros FT-IR de 3 misturas NR/SBR estudadas (20/80; 70/ $30 ; 90 / 10)$

Tabela 3. Dados FT-IR de misturas de NR/SBR com diferentes teores de elastômeros)

\begin{tabular}{cccccc}
\hline \multirow{2}{*}{ Mistura } & \multicolumn{2}{c}{$[\mathbf{N R}] /[\mathrm{SBR}]$} & $\begin{array}{c}\mathbf{A}_{\mathbf{8 8 5}} / \mathbf{A}_{699} \\
\text { (valor } \\
\text { mediano) }\end{array}$ & $\begin{array}{c}\text { Desvio } \\
\text { Médio } \\
\text { Padrão }\end{array}$ & $\begin{array}{c}\text { Desvio } \\
\text { Relativo } \\
(\%)\end{array}$ \\
\hline $10 / 90$ & 0,11 & 0,061 & 0,131 & 0,002 & 1,76 \\
$20 / 80$ & 0,25 & 0,231 & 0,207 & 0,004 & 1,95 \\
$30 / 70$ & 0,428 & 0,481 & 0,238 & 0,004 & 1,53 \\
$50 / 50$ & 1 & 0,903 & 0,445 & 0,015 & 3,46 \\
$70 / 30$ & 2,33 & 2,299 & 0,941 & 0,014 & 1,49 \\
$80 / 20$ & 4 & 4,727 & 1,538 & 0,034 & 2,24 \\
$90 / 10$ & 9 & 7,772 & 2,46 & 0,042 & 1,70 \\
\hline$=$ nominal; $\quad \mathrm{b}=$ obtido dos valores DTG de $\mathrm{h}_{1} / \mathrm{h}_{2}$ & & \\
\hline
\end{tabular}

proporção da porcentagem da composição, e, então, valores de absorvância relativa $\mathrm{A}_{885} / \mathrm{A}_{699}\left(\mathrm{~A}_{1} / \mathrm{A}_{2}\right)$, mediana $(\mu)$ de cinco (5) análises, foram calculados e colocados em função da proporção de [NR]/[SBR], para contornar problemas de variação de espessura.

$\mathrm{Na}$ Figura 4 pode-se observar que as intensidades das bandas estão relacionadas à proporção de cada componente nas misturas, como esperado pela Lei de Lambert-Bee ${ }^{[1,13]}$. A Tabela 3 apresenta os valores de $\left(A_{1} / A_{2}\right)$ em função dos dados de concentração [NR]/[SBR], obtidos pela análise DTG. Os valores de desvio médio padrão e relativo foram calculados de acordo com Hórak, e adotada em trabalhos anteriores do grupo $^{[11,14]}$.

A Figura 5 mostra a curva analítica DTG/FT-IR, ou seja, $\left(\mathrm{A}_{1} / \mathrm{A}_{2}\right)$ versus $[\mathrm{NR}] /[\mathrm{SBR}](\% \mathrm{p} / \mathrm{p})$. Da curva analítica (Tabela 3), a seguinte correlação $(\mathrm{R}=0,998)$ é proposta:

$$
\left(\mathrm{A}_{885} / \mathrm{A}_{699}\right)=0,3121[\mathrm{NR}] /[\mathrm{SBR}]+0,1092
$$

Para calcular o teor de NR e SBR, o valor mediano de $\left(\mathrm{A}_{885} / \mathrm{A}_{699}\right)$ deve ser relacionado à Equação 2 para encontrar a concentração relativa [NR]/[SBR]. Após, a Equação 3 deve ser usada:

$$
[\mathrm{NR}]+[\mathrm{SBR}]=100 \%
$$




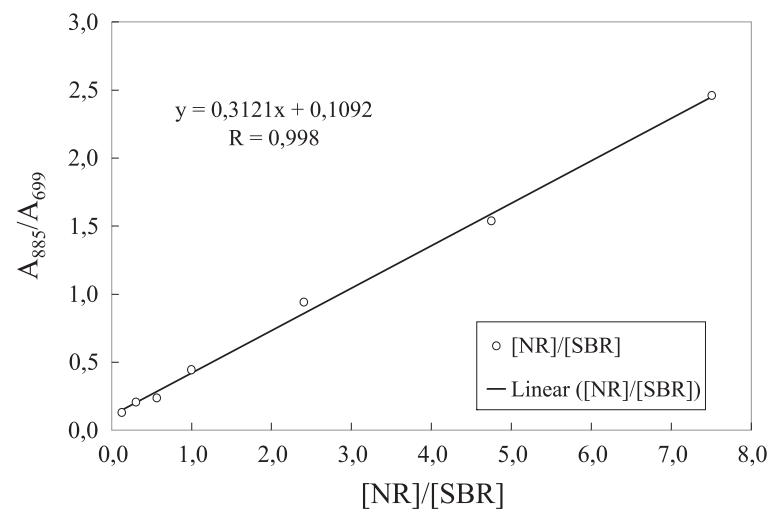

Figura 5. Curva analítica TG/FT-IR: $\left(\mathrm{A}_{1} / \mathrm{A}_{2}\right)$ versus $[\mathrm{NR}] /[\mathrm{SBR}]$

O erro relativo encontrado na metodologia $(1,73 \%)$ está dentro dos limites de precisão do espectrômetro FT-IR, $\leq 2 \%{ }^{[14]}$.

\section{Conclusões}

O uso de pirolisador com melhor controle de temperatura e da técnica DTG para o cálculo do teor real dos elastômeros nas misturas melhora a precisão da curva analítica FT-IR. Foi desenvolvido um método DTG/FT-IR para determinar o teor de elastômeros NR e SBR formulações vulcanizadas a partir de formulações onde a concentração destes elastômeros seja conhecida.

Este método é puramente quantitativo dependente de se ter disponível os elastômeros NR e SBR para a construção da curva analítica de correlação, porém independe do tipo e teor de carga presente o que o torna uma ferramenta muito útil para controle de qualidade de processo ou recebimento de matéria prima.

\section{Referências Bibliográficas}

1. Gedeon, B. J.; Ngyuen, R. H. - Rubber Division Meeting, American Chemical Society, Cleveland, Ohio, Oct 14, paper 64 (1985) .
2. Wake, W.C.; Tidd, B. K.; Loadman, M. J. R. - Analysis of Rubber and Rubber-like Polymers, third Edition, Applied Science Publishers (1983).

3. Annual Book of ASTM Standards, Part 37 ASTM D3677 (1982).

4. Dutra, R.C.L - Analyse quantitative infrarouge des associations du caoutchouc, IFOCA, Paris, (1988).

5. Sircar, A. K.; Lamond, T. G. - Rubber Chem. Technol. 48, 301, (1975).

6. Brazier, D. W. - Rubber Chem. Technol. 53, 437, (1981).

7. Sircar, A. K. - J.Sci. Ind. Res. 41, 536, (1982).

8. Lourenço, V. L.; Dutra, R. C. L.; Azevedo, M. F. P. \& Diniz, M. F. in Anais do $2^{\circ}$ Congresso Brasileiro de Polímeros, São Paulo, Vol. 1, 153, (1993).

9. Brazier, D. W. \& Nickel, G. H. - Rubber Chem. Technol. $48,661,(1975)$

10. Yang, J.; Kaliaguine, S. \& Roy, C. - Rubber Chem. Technol. 66(2), 213, (1993).

11. Dutra, R. C. L.; Lourenço, V. L.; Diniz, M. F.; Azevedo, M. F. P.; Barbosa, R. V. \& Soares, B. G. - Polymer Bulletin, 36, 593, (1996)

12. Dutra, R. C. L. \& Soares, B. G. - Polymer Bulletin, 41, 62, (1998).

13. Smith, A. L. - Applied Infrared Spectroscopy, John Wiley \& Sons, New York, (1979).

14. Hórak, M. \& Vítek, A. - Interpretation and processing of Vibrational Spectra, John Wiley \& Sons, New York, (1978).

Enviado: 08/03/04

Reenviado: $14 / 06 / 04$

Aprovado: 13/08/04 